\title{
AN RF WAVEGUIDE DISTRIBUTION SYSTEM FOR THE ILC TEST ACCELERATOR AT FERMILAB'S NML*
}

\author{
Christopher Nantista, Chris Adolphsen, Gordon Bowden, Richard Swent, and Bobby McKee, \\ SLAC, Menlo Park, CA 94025, U.S.A.
}

\begin{abstract}
An ILC R\&D facility is being constructed in the NML building at Fermilab which, in addition to an injector and beam dump with spectrometer, will contain up to three cryomodules of ILC-type superconducting 9-cell cavities. This linac will be powered by a single klystron. As part of SLAC's contribution to this project, we will provide a distribution network in WR650 waveguide to the various cavity couplers. In addition to commercial waveguide components and circulators and loads, this sytem will include adjustable tap-offs, and customized hybrids. In one configuration, the circulators will be removed to test pair-wise cancellation of cavity reflections through hybrids. The system will be pressurized with nitrogen to 3 bar absolute to avoid the need for $\mathrm{SF}_{6}$. The full distribution system for the first cryomodule will be delivered and installed later this year. We describe the design of the system and completed RF testing.
\end{abstract}

\section{INTRODUCTION}

At Fermilab, a test bed for the development of L-band $(1.3 \mathrm{GHz})$ SRF linac accelerator technology for the International Linear Collider (ILC) is being constructed. As ILC designs have evolved beyond those of the TESLA systems installed in DESY's TTF, now primarily dedicated to the FLASH VUV-FEL user facility, such a test accelerator is crucial to the ILC R\&D program. This new facility, refered to as ILCTA@NML or ILC Test Area at the New Muon Lab where it is located, will eventually house a full RF unit (three cryomodules) and so serve as an ILC string test. A similar facility, called the STF, is also being built at KEK.

The first FNAL cryomodule will be installed around the end of the year, followed by another in each of the two following years. With the three cryomodules, SLAC will then provide a $10 \mathrm{MW}$ RF source (modulator and klystron), using the ILC design choices at that time. This linac section will be complemented by an injector and beam line that provide an ILC-like beam and beam analysis capability.

In the meantime, SLAC is collaborating with Fermilab on this facility by assembling and RF processing the fundamental power couplers they need for their cavities and by building the waveguide systems for distributing RF from their existing RF sources to the cavities in the cryomodules as they are installed. We describe here our plans and progress on this latter program.

\section{LAYOUT}

The baseline RF distribution system for ILC, as described in the Reference Design Report (RDR) [1], is a linear system, in which nominally equal power is extracted for each cavity from a main distribution waveguide run through hybrid couplers with successively larger fractional couplings $(1 / 8,1 / 7, \ldots 1 / 2,1)$. This conservative approach is what was envisioned for TESLA and has been implemented in the TTF at DESY.

Our distribution system adopts an alternate approach [2] in which power for pairs of cavities is tapped off from the main waveguide and then split with 3-dB hybrids. This represents an intermediate choice between linear distribution and tree-like distribution with multiple levels of binary splitting (see [3]). The same number of power dividers and loads is needed in any case. XFEL has moved toward a similar division scheme, albeit using 3port T's rather than hybrids. There are two main advantages behind our choice of layout, which is illustrated in Fig. 1.

Firstly, it allows us to incorporate our recently developed variable tap-off (VTO), a 4-port directional coupler whose coupling can be mechanically set to any value [2]. This obviates the need for several tap-off designs. It also provides freedom in distributing power among cavities which tend to have a significant spread in sustainable gradient. This is done efficiently, in that the unused power for one pair is not terminated, but

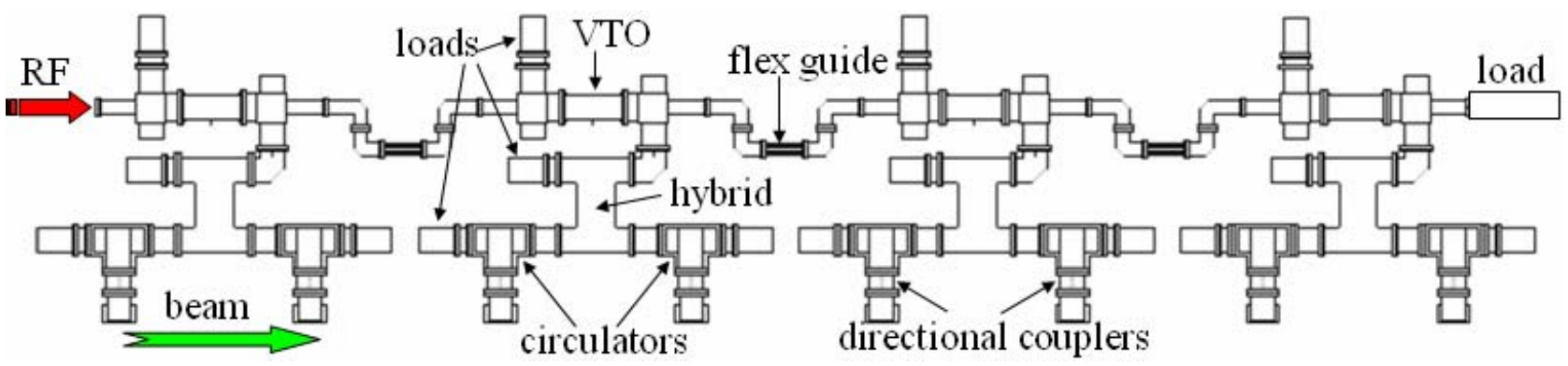

Figure 1: Layout of the WR650 RF distribution system for one cryomodule. Not shown, after U-bends at the bottom, are windows, phase shifters, and additional flexible waveguides for connecting from below into the cavity couplers.

*Work supported by the U.S. Department of Energy under contract DE-AC02-76SF00515. 
transported to the next pair. It works best if the cavities are paired in the cryomodules according to their performance in prior testing. The VTO, in its current design, is slightly too long (and perhaps too expensive) to be used for each individual cavity, but it easily fits in the space between two cavities. The customizability of power distribution within each RF unit allows the maximum sustainable gradient to be more closely approached [4], reducing the ILC cost by much more than the added cost for the VTOs.

Another advantage of our alternate approach is that by feeding the standing-wave cavities in pairs through hybrids, we can employ the standard trick whereby the power reflected from the cavities of each pair, to the extent that they present identical impedances, is combined into a load on the fourth port of their hybrid [5]. If this is shown to be sufficient to protect the klystron and isolate the cavities, the circulators can be eliminated from the ILC design. Currently included at each cavity input, these circulators are the most expensive and lossy components in the ILC RF distribution system.

Initially, as evident in Fig.1, our NML system will include circulators. Demonstrating that their elimination does not cause problems is part of the $R \& D$ program. Cancellation of reflections and phasing of the RF to the beam impose competing requirements on waveguide phase lengths (see [2]) that cannot both be met with the cavity spacing of the first two cryomodules (Type III+) to be installed. Circulators will therefore be needed to run beam. Rearrangement of the U-bends below the cavity inputs will allow us to switch between cancellation testing and beam running configurations. Testing cancellation with beam will have to await the third cryomodule, which is to be of the Type IV design with proper cavity spacing (= $53 / 4 * \lambda_{0}$ to satisfy both requirements).

Another nice feature of our distribution layout is its modularity. Each two cavity section is identical, albeit with a unique VTO setting. Four such modules will be assembled in individual support frames, wheeled into place along the accelerator, and then connected via semiflexible waveguide sections, which ease mechanical tolerances and stresses. The last module is terminated in a load to which excess power can be directed when running multiple cryomodules with the same klystron.

Another feature of note is the set of bends, or chicanes, between each two-cavity module. This fulfills two functions. It provides a simple means of adjusting the RF phase length between modules; rather than resorting to stepped waveguides with customized guide wavelength, we will simply specify the length of the vertical legs of two of the bends. There is no extra cost involved with customizing this length, whereas deviating from WR650 waveguide would require special fabrication. The other function of these chicanes is to provide a convenient way to re-adjust VTOs if necessary in-situ; removing one upper bend provides room to separate the VTO sections and rotate the center. This would be very difficult in a straight arrangement.
The WR650 flanges of our waveguide components are machined flat and connected with 1/8" metal gaskets fitted with a rubber seal on each side. Knurled inner regions on the gasket faces help provide good electrical contact. Finally, a non-standard feature of our distribution system is that most of it will be pressurized to 3 bar absolute (29 psig) with dry nitrogen. This is to hold off RF breakdown problems, particularly near the klystron window, high field points, and the circulators, as we run with peak power levels up to a few megawatts. It also provides an RF safety interlock by ensuring no open joints. Because the inputs to the cavity fundamental power couplers in the current design cannot be pressurized, we will include windows in our system, either before or after the hybrids, depending on test results of unpressurized operation at the required ILC power levels.

\section{COMPONENTS}

Our systems will incorporate a number of different waveguide components, some standard off-the-shelf parts, some reinforced with thick walls for pressurization, some developed by or for DESY for TESLA/XFEL, and some specially designed and fabricated by SLAC. We briefly describe the basic components below.

\section{Variable Tap-Off (VTO)}

The VTO is a mechanically adjustable hybrid. It works by polarization rotation, with two mode-selective end sections and a rotatable polarization-rotating middle section. This device is illustrated in Fig. 2.

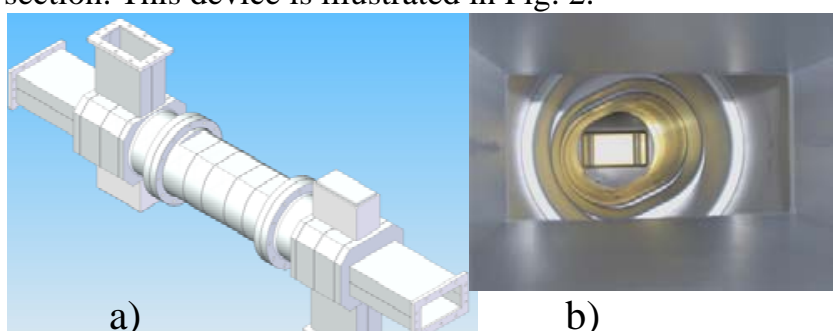

Figure 2: The VTO. a) a computer illustration and b) an interior photograph with rotated center section.

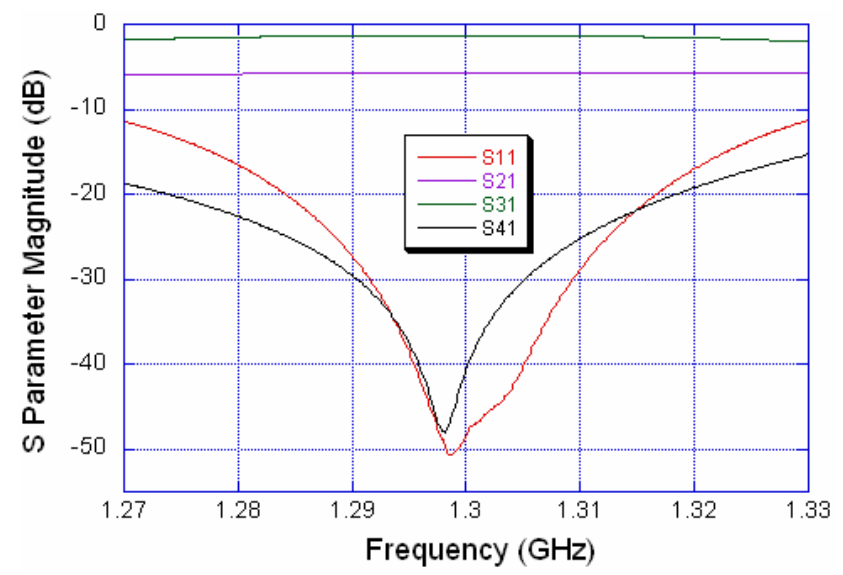

Figure 3: Network analyzer measurements of the first VTO with the coupling set at $-5.67 \mathrm{~dB}$. 
We have thus far fabricated two VTOs out of aluminum parts joined by the method of dip brazing in molten salt. Some cold-test measurements are shown in Fig. 3. Measurements were taken with the center section at four different angles, spanning the range of coupling from zero to a hundred percent. The input match or return loss varied from -48 to $-37 \mathrm{~dB}$. The phases of the through and coupled signals were stable to a fraction of a degree across the coupling range. This feature allows the phase lengths between VTOs to be set once with identical pairs of waveguide bends, independent of coupling. The measured loss in this component averaged $\sim 0.65 \%$. This could be reduced to about a half percent by copper plating the interior. High-power tests of the VTO will be performed soon.

\section{Hybrid}

Another component we decided to produce ourselves is the $3-\mathrm{dB}$ hybrid. In doing so, we hope to achieve more accurate splitting and better isolation than typically provided in commercial products. These criteria are essential to our goal of demonstrating successful operation without circulators, whereas we do not need the hybrids to be particularly broad band. Also, the orientation of the ports in our design, which is based on the "magic-H" hybrid developed at X-band [6], is a better match to our layout, requiring fewer bends. Finally, the absence of posts or sharp edges makes it more likely to be able to operate unpressurized.

The hybrid, which is pictured in Fig. 4, is fabricated by dip brazing aluminum parts as is done for the VTO. While our first try at this led to warped flanges, after re-facing them the hybrid was cold-tested and found to have excellent characteristics; the coupling measured -3.031 dB (49.8\%), the match $-43.5 \mathrm{~dB}$, the isolation $-43 \mathrm{~dB}$, and the loss $0.013 \mathrm{~dB}(0.3 \%)$. This too can be copper plated, if desired.

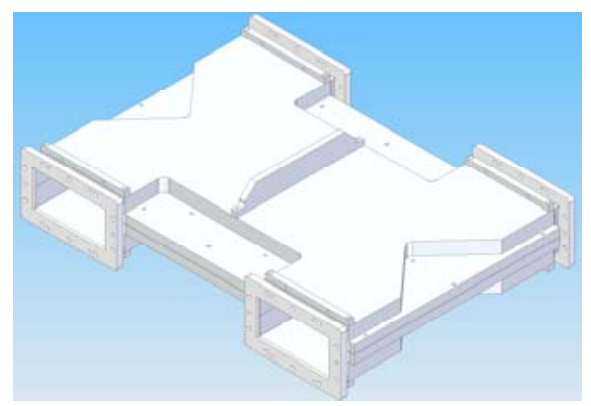

Figure 4: L-band “magic-H” hybrid.

\section{Bends and Flexible Waveguides}

Waveguide H-plane and E-plane bends are being supplied by Mega Industries, LLC. For compactness, we are using all mitered rather than swept or double-mitered bends. Our experience is that such bends do not cause problems at levels of 3-4 MW in a 3-bar pressurized system. These pieces are customized with thick walls for pressurization. Foot-long sections of semi-flexible waveguide have also been ordered, both pressurizable pieces (for the main waveguide run) and the less expensive and more flexible non-pressurizable pieces (for connection to couplers).

\section{Window}

We have a few options for the pressure windows, including a modified pillbox ceramic window from the German company Ibfm. Our preferred but as-yet untested solution is to use in-house ceramic windows of the halfwavelength dielectric plug type [7]. These are being developed and should be relatively inexpensive and no more than two inches in length.

\section{Loads and Circulator}

All high power loads for our first system are being supplied by S.P.A. Ferrite, Ltd. in St. Petersburg, Russia. They are type same as used by DESY. We use $1 \mathrm{MW}$ loads everywhere but at the end, where a $5 \mathrm{MW}$ load is installed. The circulators have built-in loads, making them isolators. For diagnostics, we also chose to use their bidirectional couplers, due to their compactness and superior directivity.

\section{Phase Shifter}

For fine-tuning the phase in each cavity, we plan to use phase shifters of the type developed at DESY and incorporated in the XFEL system [8]. It works by means of a moving side-wall, driven by a motorized feedthrough.

\section{REFERENCES}

[1] ILC Reference Design Report (soon to be available on the web).

[2] Christopher D. Nantista and Chris Adolphsen, “A Variable Directional Coupler for an Alternate ILC High-Power RF Distribution Scheme,” presented at the 2006 Linear Accel. Conf. (LINAC06), Knoxville, TN, Aug. 21-25, 2006; SLAC-PUB-12372.

[3] V. Katalev and S. Choroba, "RF Power Distributing Waveguide Systems for TESLA," presented at the XVIII Russian Part. Accel. Conf. (RUPAC02), Oct. 1-4, 2002.

[4] K.L.F. Bane, C. Adolphsen, and C. Nantista, "RF Distribution Optimization in the ILC Main Linacs,” these proceedings.

[5] Roger H. Miller, "Comparison of Standing-Wave and Traveling-Wave Structures,” invited paper at the 1986 Linear Accelerator Conference (LINAC86), Stanford CA, June 2-6, 1986; SLAC-PUB-3935.

[6] C.D. Nantista et al., "Planar Waveguide Hybrids for Very High Power RF," presented at the 1999 Particle Accelerator Conference, New York, NY, March 29April 2, 1999; SLAC-PUB-8142.

[7] Richard M. Walker, MIT Rad. Lab. Series, Vol. 9, Sec. 4-20, p. 222.

[8] V. Katalev, S. Choroba, "Waveguide Distribution System for the European XFEL," presented at the 2006 European Particle Accelerator Conference (EPAC06), Edinburgh, Scotland, June 26-30, 2006. 